\title{
Model Parameter Estimation of Humanoid Robots using Static Contact Force Measurements
}

\author{
Jos Baelemans, Pieter van Zutven and Henk Nijmeijer \\ Dynamics and Control Group, Department of Mechanical Engineering, Eindhoven University of Technology \\ P.O. Box 513, $5600 \mathrm{MB}$, Eindhoven, the Netherlands \\ Email: j.a.j.baelemans@student.tue.nl, \{p.w.m.v.zutven, h.nijmeijer\}@tue.nl
}

\begin{abstract}
Model based controllers are widely used to control motions of humanoid robots. In most cases these are based on the center of mass (CoM) model of the humanoid. For this it is important that the parameters of this model are accurately known. In this paper we contribute a method to estimate the base parameters of the full 3D CoM model of humanoid robots. This method only uses measurements of the joint angles and static contact forces for a number of postures. We also contribute a method to determine optimal stable measurement postures. The estimation method is verified in experiments on humanoid robot TUlip and a statistical analysis is performed to check the reliability of the estimated base parameters.
\end{abstract}

\section{INTRODUCTION}

Humanoid robotics is a rapidly emerging technology that is going to be used in many places in society, including households, care and cure facilities and disaster areas. To this end, humanoid robots should be capable to perform robust, versatile and energy efficient motions. Control of such motions appears to be difficult because a humanoid robot is very complex. The nonlinear, hybrid nature of its dynamics almost enforces the use of model based controllers. Many model based controllers exist [1], [2], mainly controlling the center of mass (CoM) of the biped [3], [4], [5].

Model based control inherently requires reliable knowledge about the model parameters to achieve desirable performance. Estimation of these model parameters from experimental data has been studied intensely in the field of system identification. Classical identification on robotic systems deals with rigid structures attached to a fixed base [6], [7], [8], whereas recent work shows identification of compliant, flexible robots [9], [10], [11] and floating base systems such as humanoid robots.

Dynamic identification of floating base systems, including humans and humanoid robots has been addressed in [12], [13]. It is shown that the full dynamics of a humanoid robot can be identified without joint torque data, but with contact force information. However, velocity and acceleration information about the joints and floating base are required and it appears difficult to find optimal persistently exciting motions for which the robot does not fall. In [14] the same approach is used to show that if joint torque information is available, only partial contact force information is required. A completely different identification is performed in [15] where the modularity of their biped is used to separately estimate model parameters of many different components, such as actuators, compliant drive trains and leg parts.
Besides identification of the full robot model, it is also interesting for humanoid robots to estimate the CoM model, because, as mentioned before, many control strategies focus on control of the CoM. Moreover, the equations that describe the CoM position are significantly simpler than the equations of motion of the full robot model. By definition, the CoM position does not rely on velocities or accelerations, which are often impossible to measure and estimates may be noisy or delayed. For the same reason, no information about joint torques, actuator dynamics nor friction characteristics is required to identify the CoM position.

That is why early work estimated the CoM position of humans by taking the double integral of the ground reaction forces [16]. The problem with this method is that the integrals require unknown initial conditions. Another approach identifies a relation between the frequency response of the $\mathrm{CoM}$ and center of pressure $(\mathrm{CoP})$, but this only works for periodic motions [17]. Finally, in [18], [19] the ground reaction forces are used in static experiments to estimate the CoM position of humans and humanoid robots. However, only a simple low dimensional planar model is used in multiple directions to estimate the parameters in 3D, neglecting coupling between these directions.

Therefore in this paper we identify the base parameters of the CoM model of a full 3D humanoid robot, i.e. its link masses and center of mass positions. We contribute a method to statically estimate these parameters using CoP measurements. This method is similar to [18], [19], but it takes into account coupling between the three directions of the 3D model and it only relies on the contact forces and joint angles; no velocity or acceleration information and no joint torques, actuator model or friction characteristics are required. Moreover, we contribute an algorithm to generate optimal stable static postures that guarantee persistent excitation of the measured data, necessary for reliable system identification [7], [9], but lacking in the mentioned literature. Finally, we apply these algorithms to adult-sized humanoid robot TUlip and perform statistical analysis on the estimated base parameters to check their reliability. Such analysis is missing in the mentioned literature, but we believe it is very important in order to gain trust in the identified parameters.

This paper is organized as follows. Section II describes the humanoid robot TUlip, Section III explains the identification algorithm and Section $\mathrm{V}$ gives static identification results on experimental data and a statistical analysis. 


\section{HUMANOID ROBOT TULIP}

\section{A. Hardware}

TUlip is a $125[\mathrm{~cm}]$ tall, $23[\mathrm{~kg}]$ heavy, fully autonomous, anthropomorphic adult-size humanoid robot [20]. Depicted in Fig. 1(a), TUlip has a head, a torso, two arms and two legs. The legs both have six degrees of freedom: three revolute joints are placed in each hip, one in each knee and two in both ankles. The joints are actuated by 90 [W] and 60 [W] DC motors. On each motor an incremental encoder is placed to measure the motor axis angle. The actuators drive the joints through gearboxes with ratios of 66 and 111. On each joint axis an incremental encoder is placed to measure the joint angle, which may differ from the motor angle due to flexibilities, friction or backlash in the drive train. The number of links in each leg is six, which, including the torso, brings the total number of links to thirteen as the arms and head are neglected because of the small masses involved.

\section{B. Center of Mass Model}

TUlip can be seen as a serial kinematic chain with the stand foot as base and the swing foot as end-effector, see Fig. 1(b). For this type of robots the transformation matrices can easily be derived [21]. The position and orientation of the links of the robot are defined with respect to the base coordinate frame which is located below the ankle of the stance foot. The equations for the position of the total center of mass $(\mathrm{CoM})$ of the robot are:

$$
\boldsymbol{p}_{C o M}(\boldsymbol{q})=\left[\begin{array}{lll}
x_{C o M} & y_{C o M} & z_{C o M}
\end{array}\right]^{\top}=\frac{1}{M} \sum_{i=1}^{n} m_{i} \boldsymbol{p}_{c i}^{o}(\boldsymbol{q}),
$$

where $M$ is the total mass of the robot, $\boldsymbol{q}$ are the joint angles, $m_{i}$ is the mass of link $i=1, \ldots, n$, with $n$ the total number of links and $\boldsymbol{p}_{c i}^{o}$ is the position of the center of mass of each link $i$ with respect to the base frame:

$$
\boldsymbol{p}_{c i}^{o}=\boldsymbol{p}_{i}^{o}+\boldsymbol{T}_{i}^{o} \boldsymbol{p}_{c i}^{i}
$$

with $\boldsymbol{p}_{i}^{o}$ and $\boldsymbol{T}_{i}^{o}$ the position vector and rotation matrix of link $i$ with respect to the base frame and $\boldsymbol{p}_{c i}^{i}$ the position of the center of mass of link $i$ with respect to frame $i$ :

$$
\boldsymbol{p}_{c i}^{i}=\left[\begin{array}{lll}
c_{i x} & c_{i y} & c_{i z}
\end{array}\right]^{\top},
$$

where $c_{i x}, c_{i y}$ and $c_{i z}$ are the positions of the link center of mass with respect to the link frame in $x$-, $y$ - and $z$-direction, as shown in Fig. 1(c).

\section{IDENTIFICATION ALGORITHM}

The ground projection of the CoM coincides with the center of pressure $(\mathrm{CoP})$ in the static case, so the idea of the identification algorithm is to measure the $\mathrm{CoP}$ for different static postures and fit this data to the CoM model [18], [19].

The CoM model (1) is linear in the model parameters, so it can be rewritten in the so called regressor form, which is very suitable for parameter estimation:

$$
\boldsymbol{p}_{C o M}=\boldsymbol{R}_{s}(\boldsymbol{q}) \boldsymbol{\vartheta}_{s},
$$

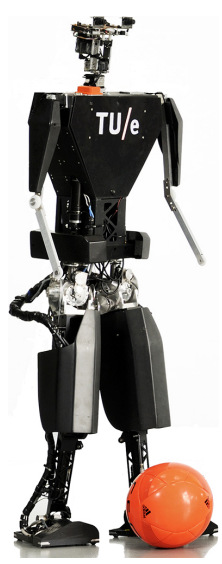

(a) Photo

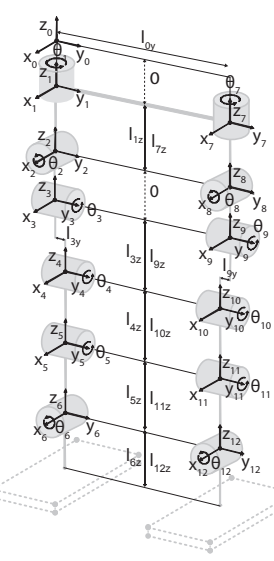

(b) Drawing of kinematics (c) Drawing of CoM model where $\boldsymbol{R}_{s}$ is the regressor matrix and $\boldsymbol{\vartheta}_{s}=\left[\begin{array}{lll}\boldsymbol{\vartheta}_{1} & \cdots & \boldsymbol{\vartheta}_{n}\end{array}\right]^{\top}$ is a vector of $4 n$ standard parameters with:

$$
\boldsymbol{\vartheta}_{i}=\left[\begin{array}{llll}
m_{i} & c_{i x} m_{i} & c_{i y} m_{i} & c_{i z} m_{i}
\end{array}\right] .
$$

The regressor form as written in (4) with (5) may have linearly dependent columns, which means $\boldsymbol{R}_{s}$ is not of full column rank so that solving (4) in a least squares sense is impossible. Hence, the standard regressor form (4) should be transformed to the so called base parameter form:

$$
\boldsymbol{p}_{C o M}=\boldsymbol{R}_{b}(\boldsymbol{q}) \boldsymbol{\vartheta}_{b} .
$$

where $\boldsymbol{\vartheta}_{b}$ is the base parameter set (BPS) and $\boldsymbol{R}_{b}$ is the base regressor matrix, which is of full column rank, i.e. all columns are linearly independent.

Rewriting (4) to (6) can be difficult especially when the equations are very large, as is the case for a humanoid robot with twelve joints. However, in [11] we have developed an automatic algorithm based on [22] to accomplish this task. Moreover, the BPS is not unique, parameters may be grouped differently to improve the final identification results. For example, the link lengths, which we assume to be known, can be put in the regressor matrix as well as the parameter vector. If put in the parameter vector, the regressor matrix only consists of trigonometric terms, so that it is better conditioned and the BPS can be solved easier.

For TUlip, the BPS consists of 27 parameters. This includes parameters in the unmeasurable $z$-direction of the CoM, but all of these parameters also appear in the $x$ - and $y$-direction, except for the vertical position of the center of mass of the feet. These parameters are only identifiable if the feet and ground are not parallel, which makes them hard to identify. However, these positions are small and assumed negligible, so the identifiable BPS consists of 25 parameters.

One static CoP measurement corresponds to two equations $\left(x_{C o M}\right.$ and $\left.y_{C o M}\right)$. There are 25 base parameters which can be derived from these equations, which means that a minimum of 13 measurements are required to solve (6) for $\vartheta_{b}$. These measurements are collected in:

$$
\boldsymbol{y}=\boldsymbol{H}(\boldsymbol{q}) \boldsymbol{\vartheta}_{b},
$$


with

$\boldsymbol{y}=\left[\begin{array}{llll}\boldsymbol{\zeta}_{1} & \cdots & \boldsymbol{\zeta}_{m}\end{array}\right]^{\top}$ and $\boldsymbol{H}=\left[\begin{array}{lll}\boldsymbol{R}_{b}\left(\boldsymbol{q}_{1}\right)^{\top} & \cdots & \boldsymbol{R}_{b}\left(\boldsymbol{q}_{m}\right)^{\top}\end{array}\right]^{\top}$,

where $\boldsymbol{\zeta}_{j}=\left[x_{C o M, j} y_{C o M, j}\right]$ and $\boldsymbol{q}_{j}$ are the joint angles, with $j=1, \ldots, m$ where $m$ is the number of measurements.

System (7) contains the base regressor form for all data points. An exact solution of (7) exists if $\operatorname{rank}(\boldsymbol{H})=$ $\operatorname{rank}\left(\left[\begin{array}{ll}\boldsymbol{H} & \boldsymbol{y}\end{array}\right]\right)$, but this is never the case because of noise, measurement errors and model inaccuracies. To average out these effects, $\boldsymbol{H}$ should contain as many measurements as possible. The solution of (7) is thus at best a fit, for example in least squares sense by minimizing $\left\|\boldsymbol{H} \hat{\boldsymbol{\vartheta}}_{b}-\boldsymbol{y}\right\|^{2}$, where $\hat{\boldsymbol{\vartheta}}_{b}$ is an estimate for $\boldsymbol{\vartheta}_{b}$. The BPS ensures that $\boldsymbol{H}$ has full column rank which means that there is a global minimum for $\left\|\boldsymbol{H} \hat{\boldsymbol{\vartheta}}_{b}-\boldsymbol{y}\right\|^{2}$, given by:

$$
\hat{\boldsymbol{\vartheta}}_{b}=\left(\boldsymbol{H}^{\top} \boldsymbol{H}\right)^{-1} \boldsymbol{H}^{\top} \boldsymbol{y} .
$$

This equation indicates that conditioning of $\boldsymbol{H}$ is important, which means that for reliable results, suitable postures need to be selected that minimize the condition number of $\boldsymbol{H}$.

\section{Static Posture Selection}

In the previous section we have seen that suitable postures are important for reliable identification results. This is especially important for 3D models as these contain significantly more parameters than planar models. In this section we explain the procedure to find suitable postures.

All robot postures have to satisfy the following constraints:

1) All joint angles $\boldsymbol{q}_{j}$ of all postures must lie within their lower $\boldsymbol{q}_{l}$ and upper $\boldsymbol{q}_{u}$ mechanical limits.

2) The robot must be stable, which means that the CoP should lie within the support polygon spanned by the feet. The support polygon is the area formed by a convex hull of the floor contact points as shown in Fig. 2. The support polygon can be computed using the kinematic model of TUlip and its foot dimensions, however, the CoM model parameters must be known. This sound contradictory, as we are trying to identify these parameters. Therefore in the posture selection procedure estimates are required for these parameters from e.g. CAD drawings. To cope with the errors in these parameters, a margin between the $\mathrm{CoP}$ and the support polygon is required. This margin is anyway advisable, since there are always errors in the joint controllers so that the desired posture is never exactly reached. Fig. 2 shows the stability margin $d_{s}$.

3) Both feet must be on the ground and they may not touch each other, so the two foot polygons, formed by the four corner points of each foot, may not intersect.

4) For the sake of convenience during the experiments, the relative position of the feet must remain the same for a number of postures. Moving the feet relative to each other means that the robot must be lifted. To decrease the experiment time, the robot moves from one posture to another with $k$ fixed feet positions.

Each static posture can be defined either in the joint space where every joint angle is specified or in the task space where

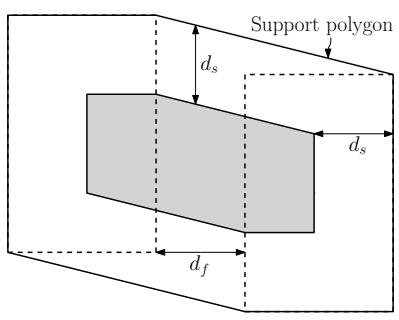

Fig. 2. Robot feet, support polygon and stability margin
TABLE I

OPTIMAL CONDITION NUMBERS

\begin{tabular}{|r|r|r|}
\hline run & \multicolumn{1}{|c|}{ Initial } & Optimal \\
\hline 1 & 647.4 & 337.5 \\
2 & 732.1 & 405.1 \\
3 & 925.8 & 352.6 \\
4 & 714.1 & 314.7 \\
5 & 733.6 & 406.9 \\
6 & 689.1 & 394.4 \\
\hline
\end{tabular}

the position and orientation of the end effectors are specified. Both methods have merits and limitations. By defining the postures in joint space, the joint limits are automatically satisfied, however, stability is not necessarily guaranteed. The opposite holds for defining the postures in task space. Either way, an optimization procedure is required, since analytical solutions cannot be found. We tried both ways and found that the optimization algorithm converges faster when the postures are defined in task space. We specify as design variables the CoM position $\boldsymbol{p}_{C o M}$, torso orientation $\phi_{T}$ and swing foot position $\boldsymbol{p}_{s}$ and orientation $\phi_{s}$. These variables constitute 9 degrees of freedom per posture, since both feet are constrained on the ground. The task space coordinates are mapped to the joint space using an inverse kinematics algorithm based on differential kinematics [21].

Initial values which are feasible within the constraints for the iterative optimization algorithm can be found as follows:

1) Choose a random position $\left(\boldsymbol{p}_{s}\right)$ and orientation $\left(\phi_{s}\right)$ of the swing foot within predefined ranges.

2) Check if the polygons which define the circumference of the feet do not intersect. If they do, go to step 1 .

3) Choose a random CoM position $\left(\boldsymbol{p}_{C o M}\right)$ within the area which is a distance $d_{s}$ from the support polygon as shown in Fig. 2. Also choose a random CoM height and torso orientation within a predefined range.

4) Solve the inverse kinematics and check if the configuration is feasible within the joint limits. If not, go to step 1 if no possible postures were found earlier for this foot placement or redo step 3 otherwise.

5) Store the feasible posture. If more postures with the same foot placement are desired, go to step 3, otherwise go to step 1 to find another foot placement, or stop if enough random postures are obtained.

Optimal postures correspond to a minimum in the condition number $\kappa$ of the output data matrix $\boldsymbol{H}$, such that we can formulate a nonlinear constrained optimization problem:

$$
\begin{gathered}
\arg \min (\kappa(\boldsymbol{H}(\boldsymbol{\chi}))), \\
\text { s.t. } \boldsymbol{q}_{j} \geq \boldsymbol{q}_{l}, \quad \boldsymbol{q}_{j} \leq \boldsymbol{q}_{u}, d_{e, j} \geq d_{s} \text { and } d_{f, j} \geq 0,
\end{gathered}
$$

where $d_{e}$ is the distance from the CoP to the edge of the support polygon, $d_{f}$ is the smallest distance between the feet (see Fig. 2) and $\chi$ are the design variables:

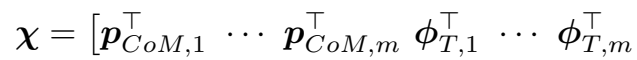

$$
\begin{aligned}
& \left.\begin{array}{llllll}
\boldsymbol{p}_{s, 1}^{\top} & \cdots & \boldsymbol{p}_{s, k}^{\top} & \boldsymbol{\phi}_{s, 1}^{\top} & \cdots & \boldsymbol{\phi}_{s, k}^{\top}
\end{array}\right]^{\top} \text {. }
\end{aligned}
$$



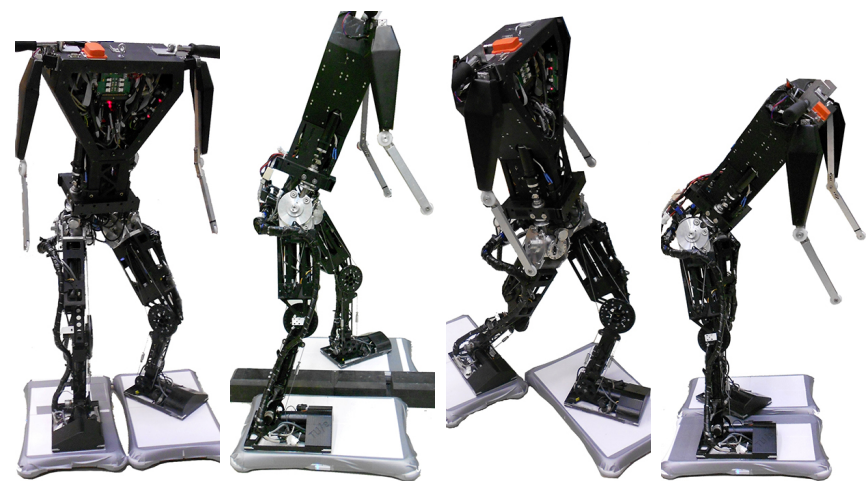

Fig. 3. Examples of optimal identification postures on TUlip

For the identification experiment, we compute sets of 50 feasible initial values, where we choose ten different foot positions with, per foot position, five different CoM positions and torso orientations. These initial values are optimized using the interior point algorithm as implemented in the Matlab Optimization Toolbox. The optimized postures may only be optimal locally, so we repeated the optimization six times for different initial values. The initial and optimized condition number for each trial are presented in Table I. As can be seen from this table the minimums vary depending on the initial values. We choose the three sets of postures with the lowest condition number for the experiments.

\section{STATIC IDENTIFICATION RESULTS}

In this section we first explain the measurement procedure and identification results, after which we validate and analyze the estimated parameters statistically.

\section{A. Measurement Procedure}

The CoP can be determined by measuring the forces between the feet and the ground. We use Wii balance boards (WBB) to measure these forces. A WBB is a $50 \mathrm{~cm}$ by 30 $\mathrm{cm}$ board, originally designed by Nintendo. These boards are equipped with a force sensor on each corner, which means that it is possible to determine the $\mathrm{CoP}$ of the object that is put on the board. A WBB is an inexpensive measurement device. Because of the size of these balance boards, we place a WBB under each foot of TUlip. The joint angles are measured using the incremental joint encoders.

Now, the measurement procedure is as follows. First, the robot is lifted and brought to the first posture, such that the WBBs can be placed in a suitable position. The robot is put with his right foot on a marked fixed point (e.g. in a corner) on a WBB and the other WBB is positioned under its left foot. Then the relative position between the WBBs is measured; it is important that the WBBs are accurately placed in the same orientation. The position of the left foot of the robot is known through the joint encoders, so that the positions of the WBBs with respect to the robot's feet can be computed, and hence, the position of the CoP with respect to the robot is known.

The robot keeps the same foot positions for five different postures so that it can stay on the WBB without being lifted.
The robot moves automatically between postures and may be supported during this movement to guarantee its stability. The robot stays in each posture for 60 seconds so that any initial motion has damped out. After five postures, the robot is lifted and its foot positions are changed, so that the WBBs need to be repositioned.

A total of $m=108$ postures appeared to be stable and feasible on the real robot. Some postures fail because of tracking errors in the joint controllers, which unbalance the robot. A few postures have been measured twice to check the measurement consistency, which appears reliable. An example of four postures can be seen in Fig. 3. For every posture, the average joint angles for the final 30 seconds are used in the regressor matrix as one data point. The first 30 seconds are omitted to guarantee that the posture is really static. With all these regressor matrices a 216 by 25 output data matrix $\boldsymbol{H}$ is constructed, since every measurement consists of the CoP position in two directions and the model contains 25 base parameters. Also the contact forces are averaged per posture for the last 30 seconds so that the CoP can be computed via:

$$
\boldsymbol{p}_{C o P}=\frac{1}{F_{s}} \sum_{i=1}^{s} F_{s, i} \boldsymbol{p}_{s, i},
$$

where $F_{s}=M g$ is the total normal force, $\boldsymbol{p}_{s, i}$ is the position of sensor $i$ with respect to the base coordinate frame and $F_{s, i}$ is the force at sensor $i$. The number of sensors is equal to $s=8$, as each WBB has four force sensors. With the CoP positions $\boldsymbol{p}_{C o P}$ for each posture, the output data $\boldsymbol{y}$ is constructed in (7). Now, a least squares estimate of the BPS $\hat{\vartheta}_{b}$ can be found using (8). The obtained BPS estimates for a full CoM model of TUlip are given in Table II.

\section{B. Validation, Statistical Analysis and Discussion}

To validate these results we compute the error between the measured and estimated $\mathrm{CoP}$ using the measured joint angles and estimated BPS:

$$
\boldsymbol{e}=\boldsymbol{y}-\hat{\boldsymbol{y}}=\boldsymbol{y}-\boldsymbol{H} \hat{\boldsymbol{\vartheta}}_{b} .
$$

The norm of this error per posture is shown in Fig. 4. Overall, this figure shows good results, which means that we can estimate the CoM position accurately. The mean, standard deviation and maximum error for the full 3D CoM model are $4.2[\mathrm{~mm}], 3.6[\mathrm{~mm}]$ and $10.7[\mathrm{~mm}]$ respectively, which is similar as with the planar decoupled models of [18], [19].

In contrast to [18], [19], we are not only interested in the results of the total CoM position, but also in the reliability of the individual base parameters. Thus, Table II also gives as precision measure the relative standard error [9], [23] of the $j^{\text {th }}$ base parameter estimate $\hat{\vartheta}_{b, j}$ :

$$
\operatorname{se}\left(\hat{\vartheta}_{b, j}\right)_{r e l}=100 \cdot \frac{\sqrt{\hat{\sigma}^{2} C_{j j}}}{\left|\hat{\vartheta}_{b, j}\right|},
$$

where $C_{j j}$ is diagonal element $j$ of $\boldsymbol{C}=\left(\boldsymbol{H}^{\top} \boldsymbol{H}\right)^{-1}$ and:

$$
\hat{\sigma}^{2}=\frac{\|\boldsymbol{e}\|^{2}}{r-p},
$$


TABLE II

PARAMETER ESTIMATION WITH EXPERIMENTAL DATA

\begin{tabular}{|r|r|r|r|r|}
\hline & \multicolumn{2}{|r|}{ Full Model } & \multicolumn{2}{|c|}{ Simplified Model } \\
\hline BPS & $\begin{array}{r}\text { Estimated } \\
\text { nr. }\end{array}$ & $\begin{array}{r}\text { Rel.std. } \\
\text { err. [\%] }\end{array}$ & $\begin{array}{r}\text { Estimated } \\
\text { [mm] }\end{array}$ & $\begin{array}{r}\text { Rel.std. } \\
\text { err. [\%] }\end{array}$ \\
\hline$\vartheta_{b, 1}$ & -10.96 & 13.0 & -11.72 & 11.2 \\
$\vartheta_{b, 2}$ & -2.34 & 245.7 & & \\
$\vartheta_{b, 3}$ & 4.83 & 170.6 & & \\
$\vartheta_{b, 4}$ & 1.55 & 765.8 & & \\
$\vartheta_{b, 5}$ & -5.87 & 297.2 & -1.34 & 129.4 \\
$\vartheta_{b, 6}$ & -14.07 & 47.3 & & \\
$\vartheta_{b, 7}$ & -30.91 & 26.9 & & \\
$\vartheta_{b, 8}$ & -3.28 & 243.5 & & \\
$\vartheta_{b, 9}$ & 51.35 & 30.2 & 3.60 & 32.9 \\
$\vartheta_{b, 10}$ & 76.39 & 1.9 & 77.41 & 1.8 \\
$\vartheta_{b, 11}$ & -8.80 & 67.2 & & \\
$\vartheta_{b, 12}$ & 44.15 & 104.6 & -11.99 & 12.7 \\
$\vartheta_{b, 13}$ & -46.83 & 99.2 & & \\
$\vartheta_{b, 14}$ & 3.68 & 137.2 & & \\
$\vartheta_{b, 15}$ & -32.62 & 88.1 & -3.02 & 32.8 \\
$\vartheta_{b, 16}$ & 26.54 & 110.0 & & \\
$\vartheta_{b, 17}$ & 130.33 & 2.2 & 135.69 & 1.2 \\
$\vartheta_{b, 18}$ & -5.78 & 65.7 & & \\
$\vartheta_{b, 19}$ & -270.08 & 0.8 & -271.53 & 0.6 \\
$\vartheta_{b, 20}$ & -255.75 & 2.2 & -256.39 & 1.1 \\
$\vartheta_{b, 21}$ & -36.09 & 23.9 & -35.78 & 11.3 \\
$\vartheta_{b, 22}$ & 0.68 & 501.3 & & \\
$\vartheta_{b, 23}$ & 39.34 & 9.1 & 48.15 & 4.5 \\
$\vartheta_{b, 24}$ & 16.45 & 16.5 & 16.83 & 11.2 \\
$\vartheta_{b, 25}$ & -10.77 & 60.2 & -10.51 & 31.2 \\
\hline & \multicolumn{5}{|c|}{} & & \\
\hline
\end{tabular}

where $r=2 m$ is the number of data points and $p$ is the number of base parameters. A practical consideration is given in [9] about the relative standard errors. A parameter is considered well identified if its relative standard error is less than 15. If this is not the case then the parameter either has no significant effect on the model or it is not excited enough by the experimental data.

The results in Table II show that approximately one third of the parameters is estimated reasonably well. Fortunately, these parameters also constitute the ones with the most influence on the CoM position since their absolute values are the highest. All parameters have unit $[\mathrm{m}]$ and the regressor only constitutes of trigonometric functions which lie in the interval $[-1,1]$. So the influence of each base parameter on the CoM position is directly coupled with its absolute value. Unfortunately, the reliability of smaller parameters, although less interesting, is in some cases relatively low.

This can partly be explained by the small influence on the CoM model, but another reason is given in [9]. As a rule of thumb, the number of data points must be at least 500 times the number of parameters. With 25 base parameters, this is not practical in the current setup. A third reason may be that the optimized postures are still too similar, due to the small constrained optimization space. This would result in (almost) linearly dependent columns in the regressor. The

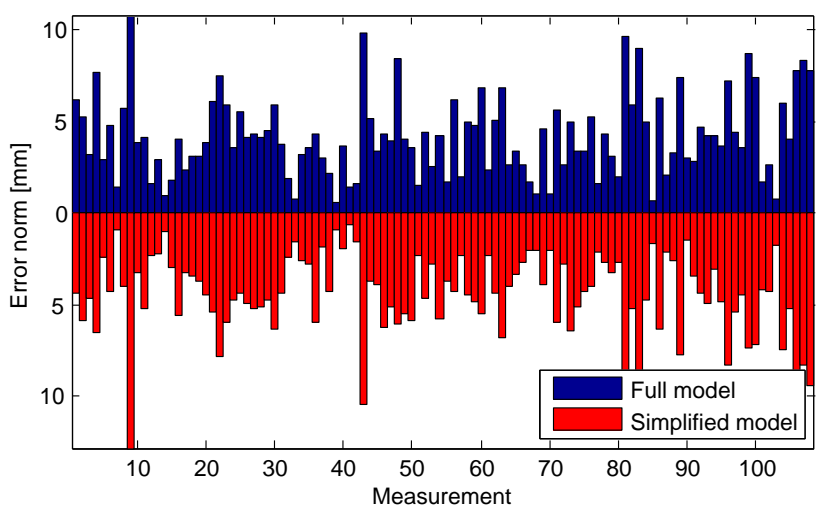

Fig. 4. Validation of identified parameters. Norm of estimation error (12) per posture, i.e. shortest distance between measured and estimated CoP.

correlation between columns $i$ and $j$ is [23]:

$$
\rho_{i j}=\frac{C_{i j}}{\sqrt{C_{i i} C_{j j}}},
$$

so that $\rho_{i j}=0$ means that parameters $i$ and $j$ are independent and $\rho_{i j}=1$ or $\rho_{i j}=-1$ means respectively a positive or negative linear relation.

The base parameters with the highest correlation are $\vartheta_{b, 12}$ with $\vartheta_{b, 13}$ and $\vartheta_{b, 15}$ with $\vartheta_{b, 16}\left(\rho_{12,13}=-0.9928\right.$ and $\rho_{15,16}=-0.9882$ ) which explains their large relative standard errors in Table II. The base parameter $\vartheta_{b, 12}$ contains the $y$-position of the right hip, upper leg, lower leg and ankle mass, whereas $\vartheta_{b, 13}$ is the $y$-position of the right foot mass. The only difference in the corresponding columns of the regressor matrix between these parameters is a multiplication with $\cos \left(\theta_{6}\right)$, which is the right ankle roll joint. This joint has a small range $\left(\theta_{6} \in[-20,17]\right.$ degrees), so that $\cos \left(\theta_{6}\right) \approx 1$ for all possible postures, which explains the strong linear relationship between these two base parameters. The left ankle roll is similar which explains the correlation between parameters $\vartheta_{b, 15}$ and $\vartheta_{b, 16}$. These base parameters are separately identifiable in theory, but the difference in the corresponding columns in the regressor matrix is too small to estimate these base parameters independently in practice. All other correlations are smaller than 0.85 .

There also seems to be a problem, although to a lesser degree with all $x$-positions of the masses of a leg. The base parameters $\vartheta_{b, 2}$ till $\vartheta_{b, 5}$ for the right leg and $\vartheta_{b, 6}$ till $\vartheta_{b, 9}$ for the left leg show large relative standard errors. The difficulty of estimation in these parameters is probably caused by the fact that the leg limbs on the robot are almost symmetrical in $x$ - and $y$-direction, so the corresponding $c_{i x}$ and $c_{i y}$ parameters are probably very small.

Possible ways to improve the estimation results include:

- Increased accuracy of CoP measurements lowers (14).

- Increased number of postures $m$ lowers (14).

- Improved optimized postures lowers the condition number of the matrix $\boldsymbol{H}$ which lowers $C_{j j}$.

For the first option more accurate measuring instruments are required such as professional force plates or 6 axis force sensors mounted in the ankle of the robot, which we do 
not have available. Increasing the number of measurements is very time consuming. A better option would be to use a dynamic parameter estimation which results in more data. However, it is difficult to find optimal persistently exciting motions for which the robot does not fall. Improving the static postures to decrease the condition number of $\boldsymbol{H}$ is difficult because of the joint limits and stability constraints. An option is to also include single support postures, although it is difficult to balance the robot on one foot.

Finally, another possible improvement is a simpler model. We derived a simplified model by taking into account symmetry in the robot links, and we use the same experimental data to estimate the following parameters:

- All mass values $m_{i}$.

- The 3D torso mass position, as it has the largest mass.

- The mass positions of the leg limbs only in $z$-direction, assuming symmetric legs in $x$ and $y$.

- The feet mass positions in $x$ - and $z$-direction, as the center of mass of a foot is in front of the ankle.

The estimates and relative standard errors (13) for this simplified model are also shown in Table II. We can see that for most parameters, the relative standard errors decrease, which indicates that this model is more reliable. The norm of the errors (12) are shown in Fig. 4. As can be seen, the simplified model gives similar results as the full model. The mean, standard deviation and maximal error are 4.5 [mm], 3.7 [mm] and 12.9 [mm] respectively. The maximal correlation (15) is 0.75 for the simplified model.

The advantage of the simplified model is that the CoM position can almost be computed as accurate as with the full model, without the problems that some base parameters cannot be estimated independently. A disadvantage is that the assumption is made beforehand that some parameters are zero, which cannot be confirmed by measurements.

\section{CONCLUSIONS}

In this paper we contribute a method to estimate the parameters of the center of mass (CoM) model of a full $3 \mathrm{D}$ humanoid robot. This method derives the base parameter set (BPS) and writes the model in the regressor form. It uses solely joint angles and contact force measurements for a number of static postures to identify the base parameters of the full 3D CoM model.

The static postures are determined by randomly selecting a number of initial postures which satisfy joint limit and stability constraints. These postures are optimized to find the minimal condition number of the data matrix used to estimate the BPS, which improves the accuracy of the estimated BPS.

We used the identification method on experimental data to estimate the model parameters of the humanoid robot TUlip. A statistical analysis is performed on the results, which shows that the estimation results look very reliable for the most important parameters, but some relatively small parameters appear difficult to estimate accurately. A simplified version of the full CoM model, taking into account symmetry in the robot links, gives similar results.

\section{REFERENCES}

[1] P. van Zutven, D. Kostić, and H. Nijmeijer, "On the stability of bipedal walking," LNAI series: Simulation, Modeling, and Programming for Autonomous Robots, pp. 521-532, 2010.

[2] P. van Zutven, D. Kostić, and H. Nijmeijer, "Foot placement for planar bipeds with point feet," in International Conference on Robotics and Automation, 2012, pp. 983-988.

[3] S. Kajita, M. Morisawa, K. Miura, S. Nakaoka, K. Harada, K. Kaneko, F. Kanehiro, and K. Yokoi, "Biped walking stabilization based on linear inverted pendulum tracking," in International Conference on Intelligent Robots and Systems, 2010, pp. 4489-4496.

[4] T. Koolen, T. de Boer, J. Rebula, A. Goswami, and J. Pratt, "Capturability-based analysis and control of legged locomotion, part 1: Theory and application to three simple gait models," The International Journal of Robotics Research, vol. 31, no. 9, pp. 1094-1113, 2012.

[5] D. Wight, E. Kubica, and D. L. Wang, "Introduction of the foot placement estimator: A dynamic measure of balance for bipedal robotics," Journal of Computational and Nonlinear Dynamics, pp. 19, 2008.

[6] M. Gautier and W. Khalil, "Identification of the minimum inertial parameters of robots," in International Conference on Robotics and Automation, 1989, pp. 1529-1534.

[7] J. Swevers, C. Ganseman, D. Tukel, J. de Schutter, and H. Van Brussel, "Optimal robot excitation and identification," Transactions on Robotics and Automation, pp. 730-740, 1997.

[8] J. Wu, J. Wang, and Z. You, "An overview of dynamic parameter identification of robots," Robotics and Computer-Integrated Manufacturing, vol. 26, no. 5, pp. 414-419, 2010.

[9] W. Khalil and E. Dombre, Modeling, identification \& control of robots. Butterworth-Heinemann, 2004.

[10] M. Rognant, E. Courteille, and P. Maurine, "A systematic procedure for the elastodynamic modeling and identification of robot manipulators," Transactions on Robotics, vol. 26, no. 6, pp. 1085-1093, 2010.

[11] P. van Zutven, D. Kostić, and H. Nijmeijer, "Parameter identification of robotic systems with series elastic actuators," in IFAC Symposium on Nonlinear Control Systems, 2010, pp. 350-355.

[12] K. Ayusawa, G. Venture, and Y. Nakamura, "Identification of the inertial parameters of a humanoid robot using unactuated dynamics of the base link," in International Conference on Humanoid Robots, 2008, pp. 1-7.

[13] —, "Symbolic proof of inertia-parameter identifiability of legged mechanisms from unactuated base-link dynamics," in IFAC Symposium on System Identification, vol. 15, no. 1, 2009, pp. 693-698.

[14] M. Mistry, S. Schaal, and K. Yamane, "Inertial parameter estimation of floating base humanoid systems using partial force sensing," in International Conference on Humanoid Robots, 2009, pp. 492-497.

[15] H. Park, K. Sreenath, J. Hurst, and J. Grizzle, "Identification of a bipedal robot with a compliant drivetrain," Control Systems, pp. 6388, 2011.

[16] O. Levin and J. Mizrahi, "An iterative model for estimation of the trajectory of center of gravity from bilateral reactive force measurements in standing sway," Gait \& Posture, vol. 4, no. 2, pp. 89-99, 1996.

[17] O. Caron, B. Faure, and Y. Brenière, "Estimating the centre of gravity of the body on the basis of the centre of pressure in standing posture," Journal of biomechanics, vol. 30, no. 11, pp. 1169-1171, 1997.

[18] S. Cotton, A. Murray, and P. Fraisse, "Estimation of the center of mass: From humanoid robots to human beings," Transactions on Mechatronics, vol. 14, no. 6, pp. $707-712$, dec. 2009.

[19] S. Cotton, M. Vanoncini, P. Fraisse, N. Ramdani, E. Demircan, A. P. Murray, and T. Keller, "Estimation of the centre of mass from motion capture and force plate recordings: A study on the elderly," Applied Bionics and Biomechanics, vol. 8, no. 1, pp. 67-84, January 2011.

[20] D. Hobbelen, T. de Boer, and M. Wisse, "System overview of bipedal robots Flame and TUlip: tailor-made for limit cycle walking," in International Conference on Intelligent Robots and Systems, 2008, pp. 2486-2491.

[21] B. Siciliano, L. Sciavicco, and L. Villani, Robotics: modelling, planning and control. Springer Verlag, 2009.

[22] H. Kawasaki, T. Shimizu, and K. Kanzaki, "Symbolic analysis of the base parameters for closed-chain robots based on the completion procedure," in International Conference on Robotics and Automation, 1996, pp. 1781-1786.

[23] D. Montgomery and G. Runger, Applied Statistics and Probability for Engineers, 5th ed. John Wiley \& Sons Inc., 2011. 